\title{
Myocardial Infarction-Associated Extracellular Vesicle-Delivered miR-208b Affects the Growth of Human Umbilical Vein Endothelial Cells via Regulating CDKN1A
}

\author{
Wenyan Jiang, ${ }^{1}$ Qiaofeng Song, ${ }^{2}$ Zhihui Lu, ${ }^{3}$ Shuo Wang, ${ }^{1}$ Tao Liu, ${ }^{1}$ Xizhu Wang, \\ and Mei Wang $\mathbb{D}^{4}$ \\ ${ }^{1}$ Department of Cardiology, Hebei Medical University, Shijiazhuang 050000, China \\ ${ }^{2}$ Department of Cardiology, Tangshan People's Hospital, Tangshan 063000, China \\ ${ }^{3}$ Department of Anesthesiology, Tangshan Workers' Hospital, Tangshan 063000, China \\ ${ }^{4}$ Department of Cardiology, The Second Hospital of Hebei Medical University, Shijiazhuang 050000, China
}

Correspondence should be addressed to Mei Wang; sciw10913@163.com

Received 22 March 2021; Accepted 24 May 2021; Published 7 June 2021

Academic Editor: Immacolata Castellano

Copyright (C) 2021 Wenyan Jiang et al. This is an open access article distributed under the Creative Commons Attribution License, which permits unrestricted use, distribution, and reproduction in any medium, provided the original work is properly cited.

\begin{abstract}
This study was aimed at investigating the effects of myocardial infarction- (MI-) associated extracellular vesicle- (EV-) delivered miR-208b on human umbilical vein endothelial cells (HUVECs). EVs were isolated and subsequently stained with PHK67. A dual-luciferase reporter gene assay was used to determine the target of miR-208b. Afterwards, HUVECs were transfected with either MI-associated EVs or miR-208b mimics, and cell viability, migration, and apoptosis were subsequently measured. Realtime quantitative polymerase chain reaction (RT-qPCR) was applied to determine the expressions of the tested genes. NanoSight, transmission electron microscopy, and western blotting showed that EVs were successfully isolated. Among the potential microRNA biomarkers for MI, miR-208b was chosen for subsequent experiments. We found that MI-associated EVs could be taken up by HUVECs and confirmed that CDKN1A was a direct target of miR-208b. Additionally, miR-208b mimics and MI-associated EVs significantly inhibited the viability and migration of HUVECs $(P<0.05)$ and promoted cell apoptosis, as well as reduced S phase and increased G2/M phase cell distribution. RT-qPCR revealed that both miR-208b mimics and MIassociated EVs upregulated the expressions of CDKN1A, FAK, Raf-1, MAPK1, and Bax but downregulated the expression of $B c l 2$ and reduced the Bcl2/Bax ratio. Our study concludes that MI-associated EVs delivered miR-208b to HUVECs, and EVdelivered miR-208b could affect the growth of HUVECs by regulating the miR-208b/CDKN1A pathway; thus, miR-208b can be therefore served as important therapeutic targets for MI treatment.
\end{abstract}

\section{Introduction}

Myocardial infarction (MI), also known as a heart attack, is caused by a decrease or cessation of blood flow to the heart, resulting in damage of the cardiac muscles due to a lack of oxygen supply [1]. The clinical symptoms of MI include heart failure, shortness of breath, arrhythmias, shock, nausea, vomiting, and chest pain [2]. Formation of plaques in the intima of arteries is one of the primary causes of MI, as the rupture of plaques can lead to myocardial ischemia and subsequent infarction [3]. With the expansion of the ageing population in China, about 1 million deaths are caused by
MI annually [4]. Currently, reperfusion therapy combined with guideline-directed medical treatment is used to treat $\mathrm{MI}$ and improve the survival of patients [3]. However, when cardiac shock occurs, the mortality rate is at least $40 \%[3,5]$. There is, therefore, an urgent need to further explore the pathogenesis of MI and identify new therapeutic targets for its treatment.

Extracellular vesicles (EVs) are released by various subtypes of cells and called exosomes, microvesicles, particles, intracellular bodies, apoptotic bodies, and many other names [6]. As carriers of proteins, lipids, and nucleic acids including DNA, RNA, and mRNA, they serve as communication tools 
among cells [7]. Previous studies have shown the usefulness of cancer cell-derived EVs in the diagnosis of many malignant tumors, such as breast cancer [8], pancreatic cancer [9], and glioblastoma [10]. Additionally, EVs also play important roles in cardiovascular disease (CVD). EVs are actually released from all cells in the cardiovascular system, and it was shown that stress conditions such as hypoxia or inflammation regulated their cargos and release with the target cells, thereby helping to improve or to impair cardiac function [11]. van Balkom et al. [12] showed that hsa-miR-214 can be delivered by endothelial cells exosomes, and transfer of this microRNA (miRNA) between endothelial cells could suppress cell senescence and promote angiogenesis. Another study indicated that miR-146a enriched in endothelial cells EVs that are efficiently taken up by cardiomyocytes had an essential role in the development of peripartum cardiomyopathy [13]. EVs isolated from stem cells of different origin have been reported to improve cardiac function after MI by reducing infarct size and fibrosis $[14,15]$. EVs produced by cardiac cells or other cells can not only be internalized by neighboring cells, and thus play roles in CVD [16], but also can be released into the body fluids and can be considered as biomarkers of CVD.

MiRNAs are the most studied elements contained in EVs, and they are short (18-25bp), noncoding RNAs that regulate protein synthesis by binding to mRNAs [17]. In the cardiovascular system, miRNAs are involved in the functional regulation of various cells, including myocardial cells, inflammatory cells, endothelial cells, smooth muscle cells, and fibroblasts, and have been proven to play an important role in CVDs [18]. A study by Agiannitopoulos et al. [19] showed that plasma miR-208b and miR-499 were significantly elevated in MI patients, proposing that they may serve as potential biomarkers for MI. Another study further suggested circulating miR-134 and miR-122-5p as potential diagnostic biomarkers for MI [20]. However, it is not clear whether MI-associated EVs, through the delivery of these miRNAs, have an impact on the development of CVD.

Since miR-499 released during MI has been investigated [21], this study determined the expression levels of miR208b, miR-122-5p, and miR-134 in the EVs isolated from the plasma of MI patients and healthy individuals. The results showed that miR-208b level in the MI-associated EVs was significantly higher than that in the normal exosomes, which indicated that miR-208b enrichment may be closely associated with the development of MI. Therefore, miR-208b was selected for the following study. Additionally, endothelial dysfunction, such as dysregulated endothelial cell proliferation, migration, and apoptosis; endothelial barrier damage; and permeability changes, is an early physiological event in CVD [22]. Therefore, human umbilical vein endothelial cells (HUVECs) were utilized to explore the effects of MI-associated EV-delivered miR-208b on HUVECs alongside their mechanisms. These findings would help improve our understanding of CVD and provide novel therapeutic targets for the prevention and treatment of MI.

\section{Materials and Methods}

2.1. Isolation of EVs from Plasma. MI patients $(n=5)$ and healthy individuals $(n=3)$ were recruited from the Department of Cardiology of Tangshan People's Hospital. Inclusion criteria for the acute MI were shown as follows: (1) typical chest pain symptoms accompanied by dynamic changes in ST-segment of electrocardiogram, (2) elevated troponin or myocardial enzyme spectrum, and (3) coronary angiography showed occlusion of at least one important coronary artery. Additionally, patients with severe cardiac insufficiency, pulmonary heart disease, severe hepatic and renal insufficiency, severe infection, rheumatism and immune system diseases, blood system diseases, and malignant tumors and those taking immunosuppressants and antitumor drugs within 24 months should be excluded. The research protocol was approved by the Ethics Committee of Tangshan People's Hospital (approval number: rmyy-llks-2020-025), and informed consent was obtained from all subjects. The physiological and biochemical indexes of all subjects are shown in Table S1.

The plasma of all subjects was collected $(5 \mathrm{~mL})$, with that of MI patients taken at 4-6 hours after onset. The samples were centrifuged at $500 \times \mathrm{g}$ at $4^{\circ} \mathrm{C}$ for $10 \mathrm{~min}$, followed by transfer of the supernatant to a new, sterile centrifuge tube for further centrifugation at $2000 \times \mathrm{g}$ at $4^{\circ} \mathrm{C}$ for $30 \mathrm{~min}$. The supernatant was collected and was centrifuged again at $10000 \times \mathrm{g}$ at $4^{\circ} \mathrm{C}$ for $30 \mathrm{~min}$. Following filtration with a 0.22 $\mu \mathrm{m}$ sterile filter, the supernatant was centrifuged at ultrahigh speed at $120000 \times \mathrm{g}$ at $4^{\circ} \mathrm{C}$ for $1 \mathrm{~h}$. Thereafter, the sediment was resuspended in $200 \mu \mathrm{L}$ sterile PBS, and the EVs were isolated and stored at $-80^{\circ} \mathrm{C}$.

2.2. Identification of Isolated EVs. The BCA protein assay kit (BOSTER Biological Technology Co., Ltd., CA, USA) was used to determine the concentration of isolated EVs, according to the manufacturer's instructions. Based on the method by Soares et al. [23], particle size distribution of EVs was measured using a NanoSight NS300 particle size analyzer (NTA, Malvern Panalytical, Malvern, UK). Thereafter, EVs were visualized by transmission electron microscopy (TEM, JEOL LTD, Peabody, MA, USA) as per a previous study [24]. Expressions of EV-specific proteins, including HSP70, CD9, and CD63, were assessed by western blotting as previously described [25]. Additionally, total RNAs from MIassociated and normal EVs were extracted using RNAiso Plus kit (Trizol, Takara Biomedical Technology Co., Ltd., Beijing, China) according to the manufacturer's protocol, and the levels of miR-208b, miR-122-5p, and miR-134 were determined using real-time quantification polymerase chain reaction (RT-qPCR). U6 was served as the housekeeping genes, and the levels of miR-208b, miR-122-5p, and miR134 were calculated using $2^{-\Delta \Delta \mathrm{Ct}}$ method [26]. The primer sequences of miR-208b, miR-122-5p, and miR-134 are displayed in Table 1.

2.3. Cell Culture and Transfection. HUVECs were purchased from Cell Bank, Chinese Academy of Sciences (Shanghai, China). The HUVECs were cultured in Dulbecco's modified 
TABLE 1: The sequences of all primers.

\begin{tabular}{|c|c|}
\hline Primer & Sequence $\left(5^{\prime}-3^{\prime}\right)$ \\
\hline hsa-miR-208b-3p-RT & GTCGTATCCAGTGCAGGGTCCGAGGTATTCGCACTGGATACGACACAAAC \\
\hline hsa-miR-208b-3p-F & GCGGCATAAGACGAACAAAAG \\
\hline hsa-miR-122-5p-RT & GTCGTATCCAGTGCAGGGTCCGAGGTATTCGCACTGGATACGACCAAACA \\
\hline hsa-miR-122-5p-F & GGCTGGAGTGTGACAATGG \\
\hline hsa-miR-134-5p-RT & GTCGTATCCAGTGCAGGGTCCGAGGTATTCGCACTGGATACGACCCCCTC \\
\hline hsa-miR-134-5p-F & GCTGTGACTGGTTGACCA \\
\hline U6-F & CTCGCTTCGGCAGCACA \\
\hline U6-R & AACGCTTCACGAATTTGCGT \\
\hline CDKN1A-hF & TGTCCGTCAGAACCCATGC \\
\hline CDKN1A-hR & AAAGTCGAAGTTCCATCGCTC \\
\hline Raf-1-hF & GGGAGCTTGGAAGACGATCAG \\
\hline Raf-1-hR & ACACGGATAGTGTTGCTTGTC \\
\hline FAK-hF & GCTTACCTTGACCCCAACTTG \\
\hline FAK-hR & ACGTTCCATACCAGTACCCAG \\
\hline Bcl-2-hF & AGTACCTGAACCGGCACCT \\
\hline Bcl-2-hR & CCACCAGGGCCAAACTGAGCA \\
\hline Bax-hF & CATATAACCCCGTCAACGCAG \\
\hline Bax $-\mathrm{hR}$ & GCAGCCGCCACAAACATAC \\
\hline MAPK1-hF & TCTGGAGCAGTATTACGACCC \\
\hline MAPK1-hR & CTGGCTGGAATCTAGCAGTCT \\
\hline GAPDH-hF & TGACAACTTTGGTATCGTGGAAGG \\
\hline GAPDH-hR & AGGCAGGGATGATGTTCTGGAGAG \\
\hline
\end{tabular}

Eagle's medium (DMEM, Thermo Fisher Scientific, Waltham, MA, USA) supplemented with 10\% fetal bovine serum (FBS, Thermo Fisher Scientific), $100 \mathrm{kU} / \mathrm{L}$ penicillin (Thermo Fisher Scientific), and $100 \mathrm{mg} / \mathrm{L}$ streptomycin (Thermo Fisher Scientific) and then incubated in $5 \%$ carbon dioxide at $37^{\circ} \mathrm{C}$.

Additionally, negative control (NC) mimics and miR208b mimics were synthesized and provided by Yanzai Biotechnology Co., Ltd., Shanghai, China. To imitate cells with miR-208b enrichment, cell transfection with NC mimics or miR-208b mimics was performed, as previously described [27]. Briefly, the HUVECs were seeded into 6-well plates $\left(5 \times 10^{5}\right.$ cells/well $)$ and then transfected with $100 \mathrm{nM}$ miR208b mimics or $100 \mathrm{nM}$ NC mimics using Lipofectamine 3000 (Thermo Fisher Scientific) based on the manufacturer's recommendations. After transfection for $6 \mathrm{~h}$, the medium was replaced with complete medium. After culturing for another $48 \mathrm{~h}$, total RNAs from the different cell groups were isolated, and the transfection efficiency was evaluated by determining the intake of miR-208b by RT-qPCR. The primer sequences of miR-208b are shown in Table 1.

2.4. Coculture of HUVECs and EVs. The EVs were labeled using the PKH67 staining kit (Sigma-Aldrich, USA) according to the manufacturer's instructions. Briefly, $60 \mu \mathrm{g}$ EVs were mixed with $970 \mu \mathrm{L}$ Diluent $\mathrm{C}$ and $4 \mu \mathrm{L}$ PKH67, incubated for $5 \mathrm{~min}$, and mixed with $1 \mathrm{~mL} \mathrm{1 \%}$ bovine serum albumin (BSA, Sigma-Aldrich) to terminate dyeing. The mixture was then centrifuged at $120000 \times \mathrm{g}$ for $60 \mathrm{~min}$, and the sediment was resuspended in $200 \mu \mathrm{L}$ PBS.
The HUVECs were seeded and cultured overnight in 24well plates $\left(5 \times 10^{4}\right.$ cells/well $)$. The next day, the cells were rinsed 3 times with serum-free medium and then incubated in the same medium. Subsequently, $15 \mu \mathrm{L}$ PKH67-labeled EVs were added, and the cells were cultured in an incubator with $5 \%$ carbon dioxide at $37^{\circ} \mathrm{C}$ for $24 \mathrm{~h}$ and $48 \mathrm{~h}$. After $3 \mathrm{PBS}$ washes, the HUVECs were fixed with $4 \%$ paraformaldehyde (Beyotime Biotechnology, Shanghai, China), rewashed with PBS, and mounted in a DAPI-containing medium (Vector Laboratories, USA). The cells were eventually observed using a laser scanning confocal microscope (Leica Microsystems, Inc., USA).

2.5. Dual-Luciferase Reporter Gene Assay. The online analytic tool, TargetScan Human 7.1 (http://www.targetscan.org/ vert_71/), was used to predict the target gene of miR-208b. The cyclin-dependent kinase inhibitor $1 \mathrm{~A}(\mathrm{CDKN} 1 \mathrm{~A}) 3^{\prime}$ untranslated region ( $3^{\prime}$ UTR) sequence (Yanzai Biotechnology Co., Ltd., Shanghai, China) and the pGL3-basic vector (Yanzai Biotechnology Co., Ltd., Shanghai, China) were combined to establish the $3^{\prime} \mathrm{UTR}$ CDKN1A reporter plasmid (pGL3-CDKN1A). Briefly, $0.3 \mu \mathrm{g}$ pGL3-basic vector and 0.3 $\mu \mathrm{g}$ pGL3-CDKN1A were cotransduced into 293T cells containing either $100 \mathrm{nM}$ miR-208b mimics or $100 \mathrm{nM} \mathrm{NC}$ mimics by using Lipofectamine 3000 (Thermo Fisher Scientific) following the manufacturer's protocol. Luciferase reactivity was then assessed using a dual-luciferase reporter system (Promega, Madison, WI, USA). 
2.6. Cell Viability and Migration Assays. The HUVECs were seeded into 96-well plates $\left(1 \times 10^{4}\right.$ cells/well $)$ and were divided into 4 groups: the control, miR-NC, miR-208b mimic, and MI-associated EV group. Cells of the miR-NC and miR-208b mimic groups were transfected with NC mimics and miR-208b mimics, respectively, while those of the control and $\mathrm{MI}$-associated $\mathrm{EV}$ groups were treated with equal PBS and MI-associated EVs $(50 \mu \mathrm{g} / \mathrm{mL}, 100 \mu \mathrm{g} / \mathrm{mL}$, and $200 \mu \mathrm{g} / \mathrm{mL}$ ), respectively. After culturing for $24 \mathrm{~h}, 48 \mathrm{~h}$, and $72 \mathrm{~h}, 10 \mu \mathrm{L}$ Cell Counting Kit-8 reagent (CCK-8, Beyotime Biotechnology) was added to each well, and the cells were incubated for $2 \mathrm{~h}$ after which the absorbance was measured at $450 \mathrm{~nm}$ using a microplate reader.

HUVEC migration of the different treatment groups was evaluated using Transwell chambers (pore size $8 \mu \mathrm{m}$; Guangzhou Jet Bio-Filtration Co., Ltd., Guangzhou, China). Harvested cells were added into the upper Transwell chambers $\left(1 \times 10^{4}\right.$ cells/well), while the lower Transwell chambers contained $500 \mu \mathrm{L}$ complete medium. After incubation for $24 \mathrm{~h}$, the chambers were removed, and the cells were washed with PBS. The cells were then fixed with $500 \mu \mathrm{L} 4 \%$ paraformaldehyde for $20 \mathrm{~min}$, washed with PBS 3 times, and stained with $0.5 \%$ crystal violet (Beyotime Biotechnology, Shanghai, China) for $20 \mathrm{~min}$. After washing and air-drying, the images were observed under a microscope, and the relative number of cell migration was analyzed.

2.7. Cell Apoptosis and Cell Cycle Assays. Cells of the different treatment groups were evaluated for apoptosis using the Annexin V-FITC apoptosis assay kit (Beyotime Biotechnology) following the manufacturer's protocol. Briefly, the cells were centrifuged at $1000 \times \mathrm{g}$ for $5 \mathrm{~min}$ and were then resuspended in $195 \mu \mathrm{L}$ Annexin V-FITC binding solution. Subsequently, $5 \mu \mathrm{L}$ Annexin V-FITC and $10 \mu \mathrm{L}$ propidium iodide (PI) staining solutions were added. After incubation in the dark for $20 \mathrm{~min}$, the cells were acquired by flow cytometry, and the apoptosis rate was calculated using the CellQuest software (Becton, Dickinson and Company, NJ, USA).

For the cell cycle assay, cells of each treatment group were centrifuged at $1000 \times \mathrm{g}$ for $5 \mathrm{~min}$ and were then resuspended in $200 \mu \mathrm{L}$ PBS. Afterwards, $4 \mathrm{~mL} 70 \%$ ethanol precooled to $-20^{\circ} \mathrm{C}$ was added, and the cells were fixed in a $4^{\circ} \mathrm{C}$ refrigerator overnight. The fixed cells were then centrifuged at $1000 \times \mathrm{g}$ for $5 \mathrm{~min}$ and subsequently resuspended in PBS with 50 $\mu \mathrm{g} / \mathrm{mL}$ RNase A. After incubation at $37^{\circ} \mathrm{C}$ for $30 \mathrm{~min}, 20 \mu \mathrm{L}$ PI was added, and the mixture was incubated in the dark for another $30 \mathrm{~min}$. Finally, a flow cytometer was utilized to observe cell cycle distribution.

2.8. RT-qPCR. The total RNA of each cell group was extracted using the RNAiso Plus kit (Trizol, Takara Biomedical Technology Co., Ltd., Beijing, China) according to the manufacturer's protocol. Thereafter, the extracted RNA was reverse transcribed into cDNA by using the PrimeScript ${ }^{\mathrm{TM}}$ II 1st Strand cDNA Synthesis kit (Takara, Dalian, China). RT-qPCR was initiated at $95^{\circ} \mathrm{C}$ for $2 \mathrm{~min}$, followed by 40 cycles at $95^{\circ} \mathrm{C}$ for $15 \mathrm{~s}$ and at $60^{\circ} \mathrm{C}$ for $60 \mathrm{~s}$. The sequences of all primers are shown in Table 1. Glyceraldehyde-3phosphate dehydrogenase (GAPDH) was used as the house- keeping gene, and the relative mRNA levels of CDKN1A, Raf-1 proto-oncogene, serine/threonine kinase (Raf-1), focal adhesion kinase $(F A K)$, BCL2 apoptosis regulator $(B c l 2)$, BCL2-associated X, apoptosis regulator (Bax), and mitogenactivated protein kinase 1 (MAPK1) were calculated using the $2^{-\Delta \Delta \mathrm{Ct}}$ method [26].

2.9. Statistical Analysis. Data are expressed as mean \pm standard deviation (SD). One-way analysis of variance (ANOVA) followed by Bonferroni method was performed via GraphPad Prism 5 (GraphPad Software, San Diego, CA) to analyze the statistical significance between more than 2 groups, whereas the Student $t$-test was used for comparisons between 2 groups. $P<0.05$ was considered statistically significant.

\section{Results}

3.1. Identification of EVs. EVs were isolated from the plasmas of MI patients and healthy individuals and were then identified by TEM, NTA, and western blotting. On TEM, the EVs displayed a nearly round- or cup-shaped morphology (Figure 1(a)). NTA analysis showed that the major peak of the isolated particles was about $55 \mathrm{~nm}$, and the overall size distribution ranged from 50-200 nm (Figure 1(b)), which was in accordance with the size distribution of EVs previously reported [28]. In terms of western blotting, all EVspecific markers, HSP70, CD9, and CD63, were expressed (Figure 1(c)). These results altogether suggested that EVs were successfully isolated.

3.2. Screen of miRNAs and Cell Transfection Efficiency. miR208b, miR-122-5p, and miR-134 have been reported to be closely associated with MI; and their levels in the EVs isolated from MI patients and healthy individuals were hence assessed using RT-qPCR. miR-208b amount in the MIassociated EVs was significantly higher than that in the controls $(P<0.05$, Figure $2(\mathrm{a}))$, while the amounts of miR$122-5 \mathrm{p}$ and miR-134 were significantly lower $(P<0.05$, Figures 2(b) and 2(c)). Furthermore, levels of miR-208b, miR-122-5p, and miR-134 were further measured in both normal HUVECs and those transfected with MI-associated EVs. No significant differences in miR-122-5p and miR-134 levels were shown between the two groups $(P>0.05$, Figures 2(e) and 2(f)), whereas miR-208b level in the EVtransfected group was significantly enriched compared with that in the normal HUVECs $(P<0.05$, Figure $2(\mathrm{~d}))$. These results indicated the ability of MI-associated EVs in delivering miR-208b to cells. Therefore, miR-208b was selected for the rest of our study.

In addition, miR-208b-enrichment cells were imitated by miR-208b mimic transfection, and the transfection efficiency was evaluated in terms of miR-208b content in the cells. No significant differences in miR-208b content were observed between the control and NC mimic groups $(P>0.05$, Figure $2(\mathrm{~g}))$. In contrast, a significant increase in miR-208b content was demonstrated in the miR-208b mimic group compared with the control group $(36509.09 \pm 2305.34$ vs. $1.03 \pm 0.31$, respectively, $P<0.05$, Figure $2(\mathrm{~g}))$. These results 


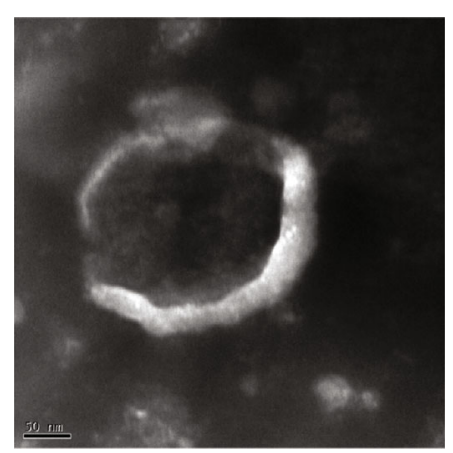

(a)

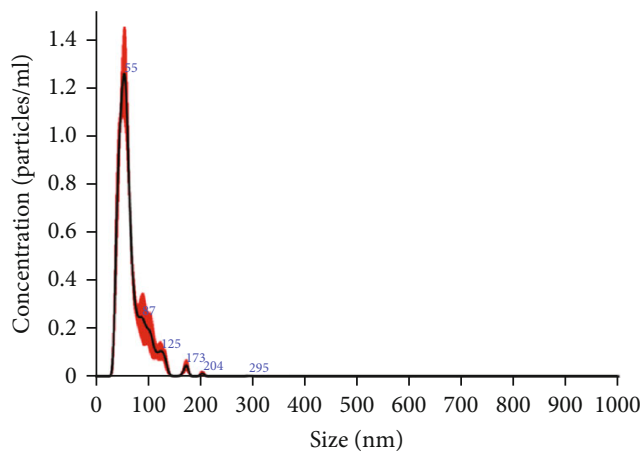

(b)

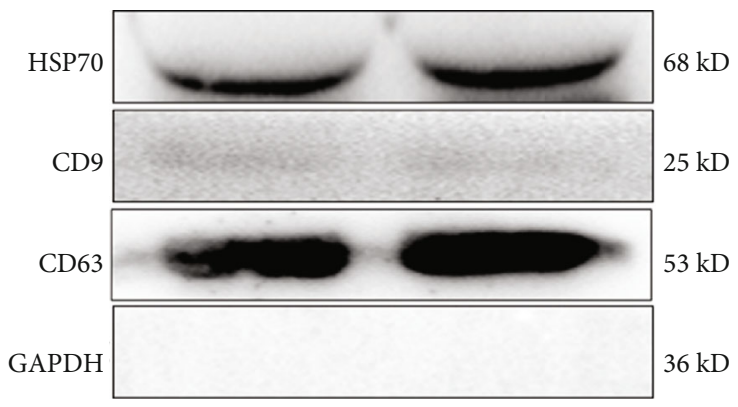

(c)

FIGURE 1: Identification of extracellular vesicles (EVs) isolated from the plasmas of myocardial infarction (MI). (a) The morphology of EVs observed by transmission electron microscopy. (b) The particle size distribution of EVs detected using the NanoSight NS300 particle size analyzer. (c) The surface markers of EVs (HSP70, CD9, CD63) were determined by western blotting, and the experiment was repeated twice $(n=2)$. Band 1 and band 2 are two duplicates.

indicated that HUVEC lines with enriched miR-208b were successfully established.

3.3. Cellular Uptake of EVs in HUVECs. MI-associated EVs were stained green by PKH67, and the nuclei of HUVECs were stained blue by DAPI. After coculture for $24 \mathrm{~h}$ and 48 $h$, it was found that most HUVECs exhibited intracellular green fluorescence (Figure 3(a)), and the fluorescence intensity after $48 \mathrm{~h}$ was significantly stronger than that after $24 \mathrm{~h}$ $(P<0.05$, Figure 3(b)). This indicated that MI-associated EVs could be taken up by HUVECs and that cell uptake of EVs increased with culture time.

3.4. miR-208b Directly Binds to CDKN1A. The online analysis tool TargetScan Human 7.1 predicted $C D K N 1 A$ as a potential target gene of miR-208b, as several miR-208b binding sites were found in the CDKN1A $3^{\prime}$ UTR (Figure 4(a)). A dual-luciferase reporter gene assay was performed to confirm this. The relative luciferase activity of pGL3-CDKN1A was significantly lower after transfection with miR-208b-3p mimics than with miRNA-NC $(P<0.05$, Figure $4(\mathrm{~b}))$. Additionally, no significant difference in relative luciferase activity was observed among pGL3-CDKN1A transfected with miRNA-NC, pGL3-basic transfected with miR-208b-3p mimics, and pGL3-basic transfected with miRNA-NC $(P>0.05$, Figure $4(\mathrm{~b}))$. These results confirmed $C D K N 1 A$ as the target gene of miR-208b.
3.5. Analyses of Cell Viability and Migration. CCK-8 was used to determine the viability of HUVECs following the different treatments. No significant difference in cell viability was observed between the control and miR-NC groups (Figure 5(a)). After the $24 \mathrm{~h}$ culture, viability of the $50 \mu \mathrm{g} / \mathrm{mL}$ MI-associated EV group was lower than that of the control group, but there was no significant difference $(P>0.05$, Figure 5(a)). After the $24 \mathrm{~h}$ and $48 \mathrm{~h}$ culture, viabilities of the miR-208b mimics and the $100 \mu \mathrm{g} / \mathrm{mL}$ and $200 \mu \mathrm{g} / \mathrm{mL}$ MI-associated EV groups were significantly reduced compared to those of controls $(P<0.05$, Figure 5(a)). However, after the $72 \mathrm{~h}$ culture, viabilities of the $50 \mu \mathrm{g} / \mathrm{mL}$ and 100 $\mu \mathrm{g} / \mathrm{mL}$ MI-associated EVs groups were significantly higher than those of the control group. Thus, HUVEC treatment with $100 \mu \mathrm{g} / \mathrm{mL}$ MI-associated EVs at a duration of $24 \mathrm{~h}$ was chosen for subsequent experiments.

Thereafter, the effects of miR-208b and MI-associated EVs on cell migration were evaluated using the Transwell assay. Mean cell numbers of the control and miR-NC groups were $316 \pm 4.5$ and $306 \pm 6.4$, respectively, which showed no significant difference $(P>0.05$, Figure 5(b)). After transfection with miR-208b mimics and MI-associated EVs, the number reduced to $256 \pm 9.7$ and $281 \pm 12$, respectively, with that of the miR-208b mimic group being significantly higher than that of the MI-associated EV group $(P<0.05$, Figure 5(b)). Results of both the CCK-8 and Transwell assays showed that miR-208b and MI-associated EVs inhibited both HUVEC viability and migration. 


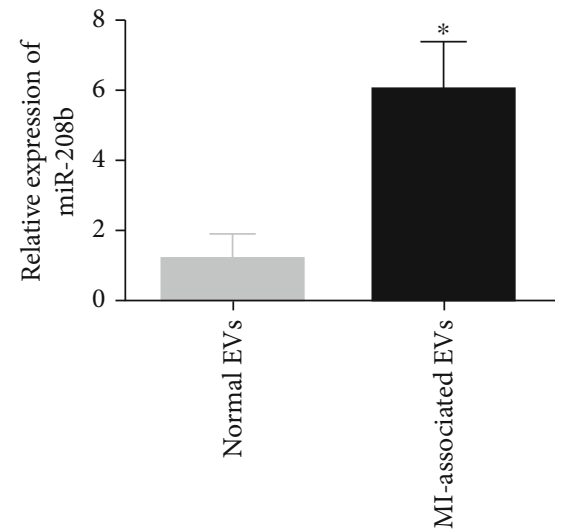

(a)

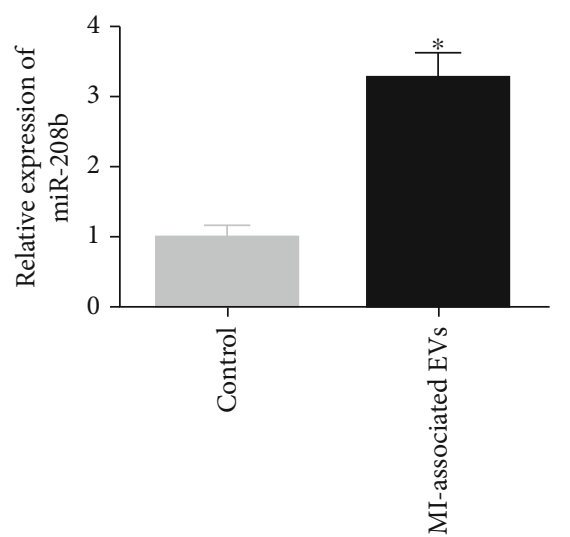

(d)

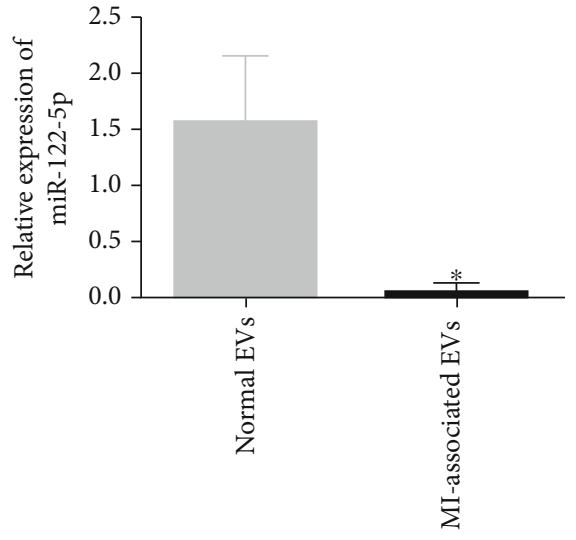

(b)

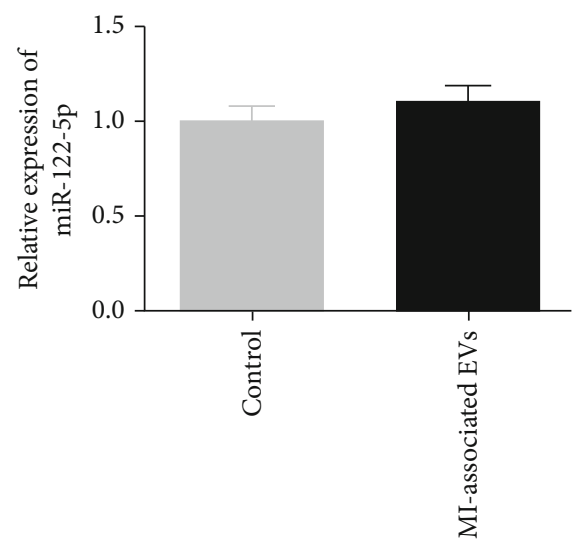

(e)

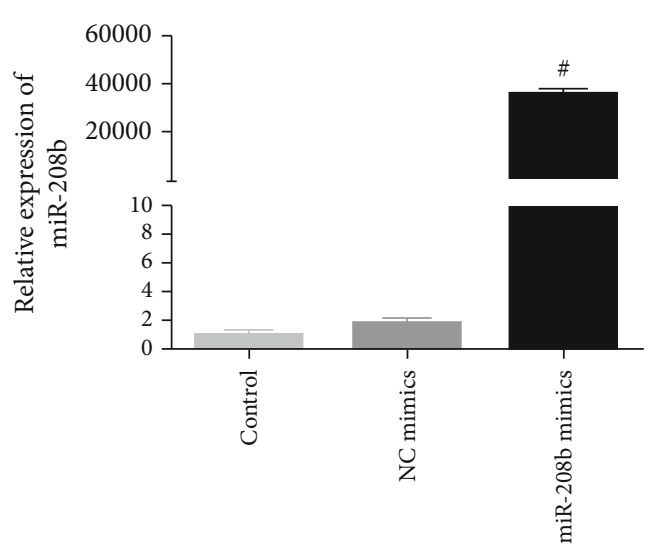

(g)

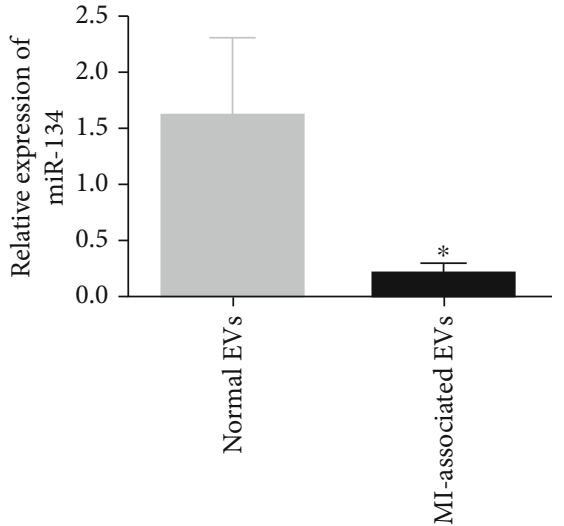

(c)

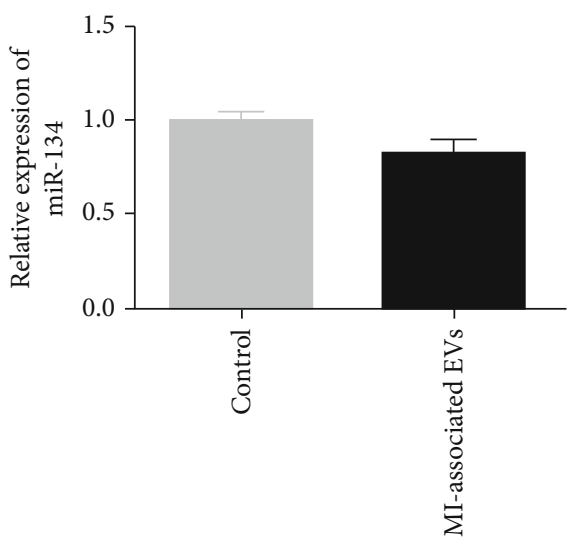

(f)

Figure 2: The levels of miR-208b (a), miR-122-5p (b), and miR-134 (c) in the EVs isolated from the plasmas of MI patients and healthy individuals, detected by real-time quantitative PCR (RT-qPCR). Normal EVs: EVs isolated from the plasma of the healthy individuals; MIassociated EVs: EVs isolated from the plasma of the MI patients. ${ }^{*} P<0.05$, compared with normal EVs, $n=3$. The levels of miR-208b (d), miR-122-5P (e), and miR-134 (f) in the control and MI-associated EV-transfected HUVECs. Control: HUVECs without treatment; MIassociated EVs: HUVECs transfected with MI-associated EVs. ${ }^{*} P<0.05$, compared with the control group, $n=3$. (g) The cell transfection efficiency evaluated based on the level of miR-208b. ${ }^{\#} P<0.05$, compared with the control group, $n=3$.

3.6. Analyses of Cell Apoptosis and Cycle. The effects of miR$208 \mathrm{~b}$ and MI-associated EVs on cell apoptosis and cycle progression were investigated by flow cytometry. Compared with the control group, apoptosis rates of both the miR$208 \mathrm{~b}$ mimic and MI-associated EV groups were significantly higher $(P<0.05$, Figure $6(a))$, with those of the miR-208b mimic group higher than those of the MI-associated EV group. Results of the cell cycle analysis showed no significant difference in cell distribution of the G0/G1 phase among the groups (Figure 6(b)). After transfection with miR-208b mimics and MI-associated EVs, the number of cells in the $\mathrm{S}$ phase was lower compared to controls, but it was higher in the G2/M phase (Figure 6(b)). Besides, the change trend after miR-208b mimic transfection was more significant compared 


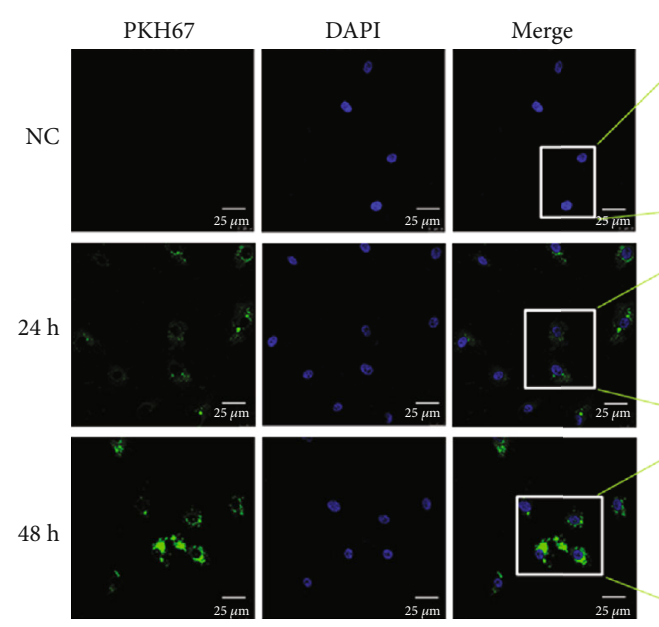

(a)
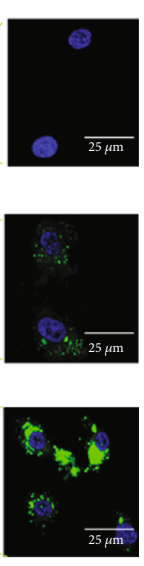

$\frac{1}{25 \mu \mathrm{m}}$

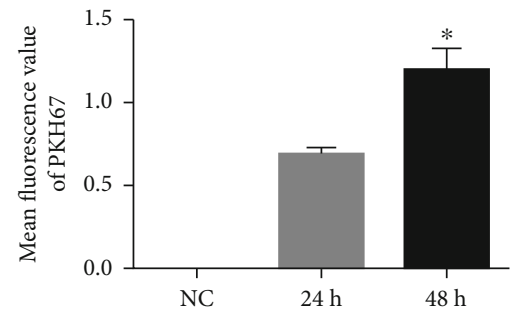

(b)

Figure 3: PKH67-labeled MI-associated EVs (green) taken up by HUVECs after coculture for $24 \mathrm{~h}$ and $48 \mathrm{~h}$. (a) Immunofluorescence images were obtained using a laser scanning confocal microscope (at the magnification of 400x). (b) Mean fluorescence values of PKH67 analyzed by ImageJ software. ${ }^{*} P<0.05$, compared with cultured for $24 \mathrm{~h}, n=3$.
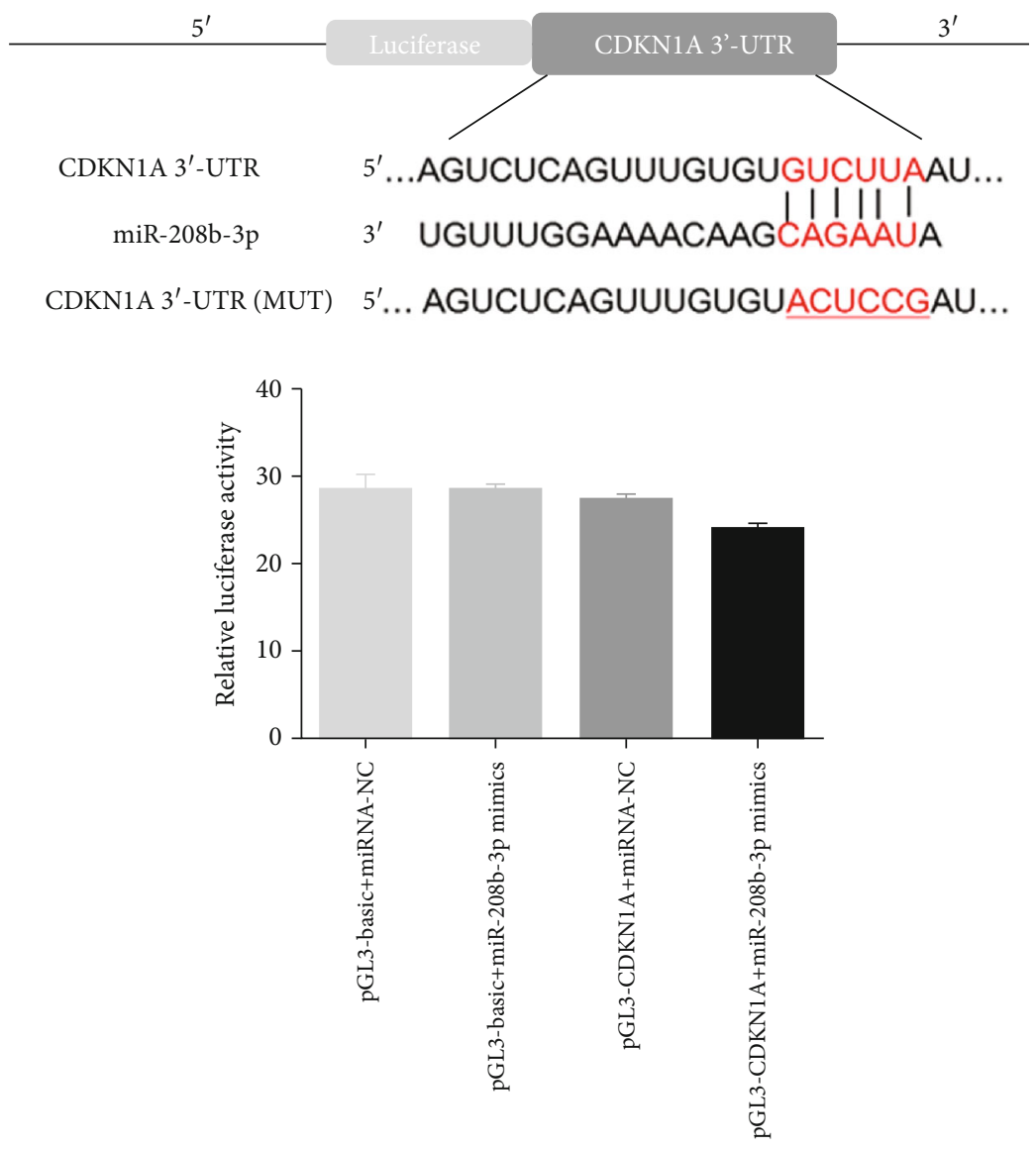

Figure 4: CDKN1A was the target gene of miR-208b. (a) TargetScan Human 7.1 was utilized to predict the target gene of miR-208b. (b) The relative luciferase activity was measured after $293 \mathrm{~T}$ cells were cotransfected with either miR-208b-3p mimics or miRNA-NC and pGL3CDKN1A luciferase vector or pGL3-basic vector, $n=3 .{ }^{*} P<0.05$, compared with the pGL3 - CDKN1A + miRNA - NC group.

with that after MI-associated EV treatment $(P<0.05$, Figure 6(b)). The results indicated that miR-208b and MIassociated EVs promoted cell apoptosis, as well as decreased $\mathrm{S}$ phase and increased $\mathrm{G} 2 / \mathrm{M}$ phase cell distribution.
3.7. RT-qPCR Analysis. In order to understand the molecular mechanisms to which miR-208b and MI-associated EVs impact HUVEC growth, RT-qPCR was employed to determine the relative expression levels of CDKN1A, Raf-1, FAK, 

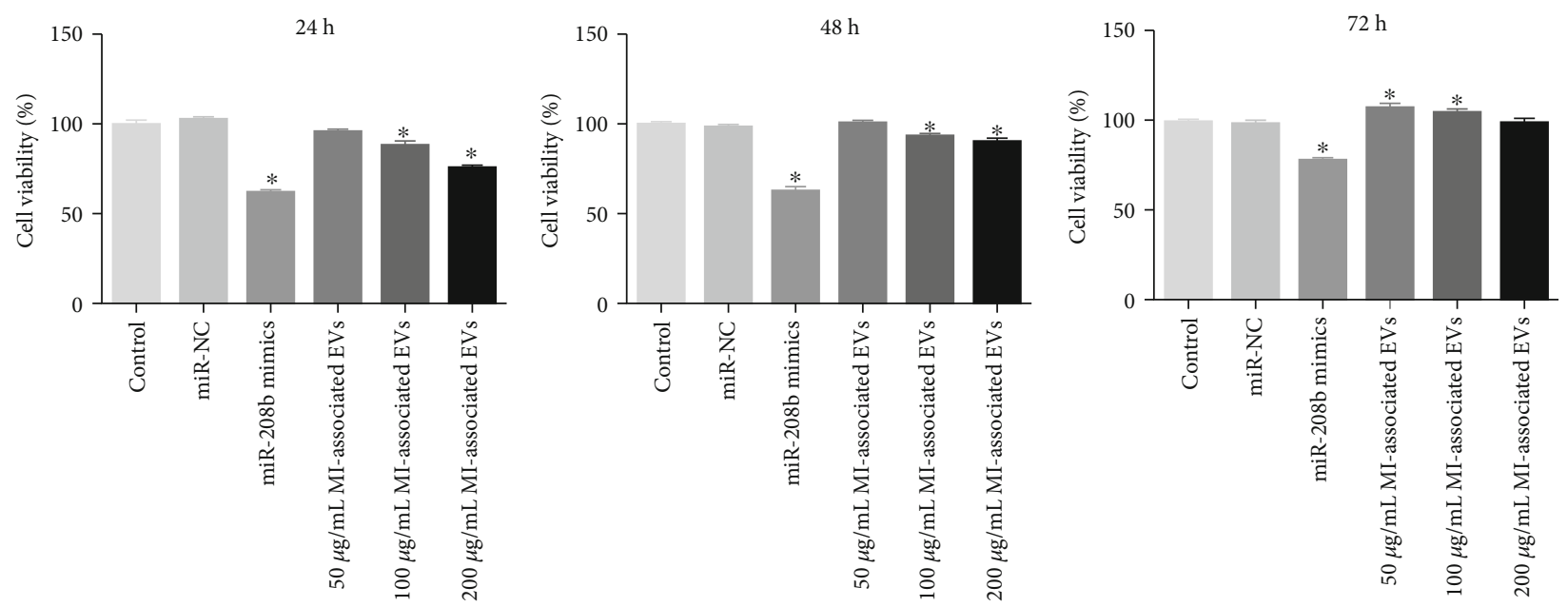

(a)

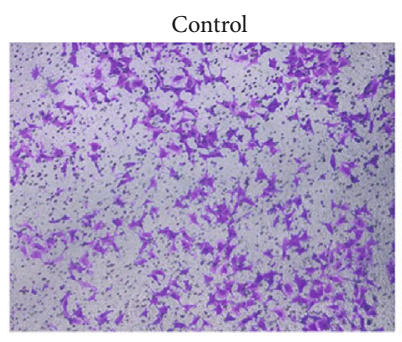

miR-208b mimics

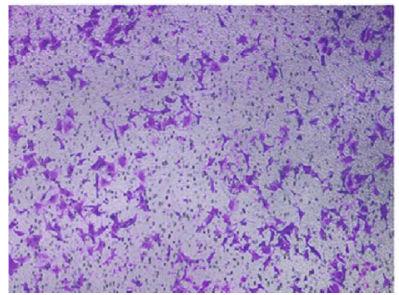

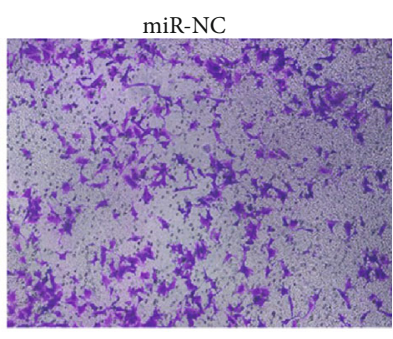

MI-associated EVs

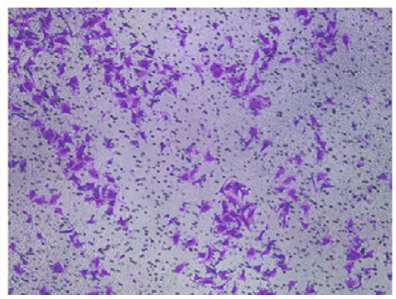

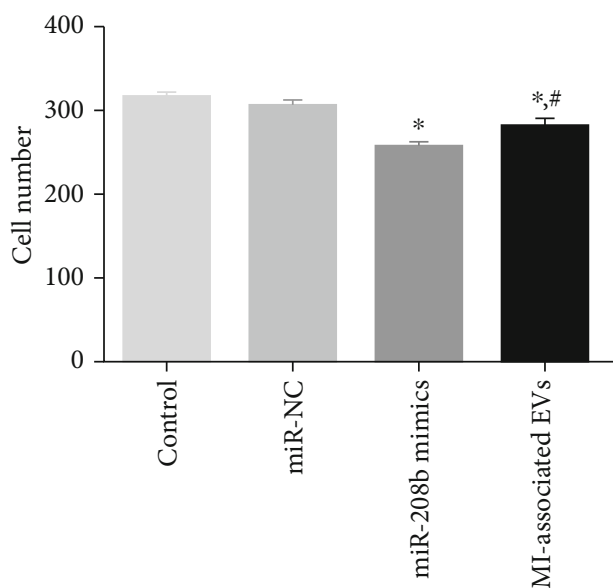

(b)

Figure 5: (a) The viability of HUVECs following the different treatments for $24 \mathrm{~h}, 48 \mathrm{~h}$, and $72 \mathrm{~h}$, determined by Cell Counting Kit- 8 (CCK8), $n=3$. (b) HUVECs were treated with $100 \mu \mathrm{g} / \mathrm{mL}$ MI-associated EVs for $24 \mathrm{~h}$, and the migration of HUVECs following the different treatments was determined by the Transwell assay. Left: images showing crystal violet staining under a microscope (at the magnification of 100x). Right: cell number of the different groups, $n=3 .{ }^{*} P<0.05$, compared with the control group. ${ }^{\#} P<0.05$, compared with the miR$208 \mathrm{~b}$ mimic group.

$B c l 2, B a x$, and MAPK1. No significant differences were demonstrated in the expression levels of all tested genes between the control and miR-NC groups. In both the miR-208b mimic and MI-associated EV groups, expression levels of $C D K N 1 A$ and $F A K$ were significantly upregulated compared with the control group $(P<0.05$, Figures $7(\mathrm{a})$ and $7(\mathrm{~b}))$. Expressions of Raf-1 and MAPK1 were also significantly upregulated $(P<0.05)$, with significantly higher expression levels in the miR-208b mimic group than in the MIassociated EV group $(P<0.05$, Figures $7(\mathrm{c})$ and $7(\mathrm{~d}))$. In terms of $B c l 2$, expression levels were significantly downregulated in the miR-208b mimic and MI-associated EV groups $(P<0.05$, Figure $7(\mathrm{e}))$; however, an opposite trend was shown for Bax expression (Figure 7(f)). The Bcl2/Bax ratio was subsequently calculated, and significantly lower values were observed in both the miR-208b mimic and MI- associated EV groups, compared to the control group $(P<0.05$, Figure $7(\mathrm{~g}))$.

\section{Discussion}

MI caused by myocardial ischemia is a major cause of morbidity and mortality worldwide, seriously affecting people's health and quality of life [29]. EVs delivered miRNAs, which act as endocrine, autocrine, and paracrine factors, have been reported to participate in the progression of diseases by mediating intercellular communication [30]. Our study is the first to explore the effects of MI-associated EVs delivered miRNAs on HUVECs. By comparing the levels of miR-208b, miR-122-5p, and miR-134 between MI-associated and normal EVs, miR-208b was found to be enriched in the MIassociated EVs and was hence selected for further 

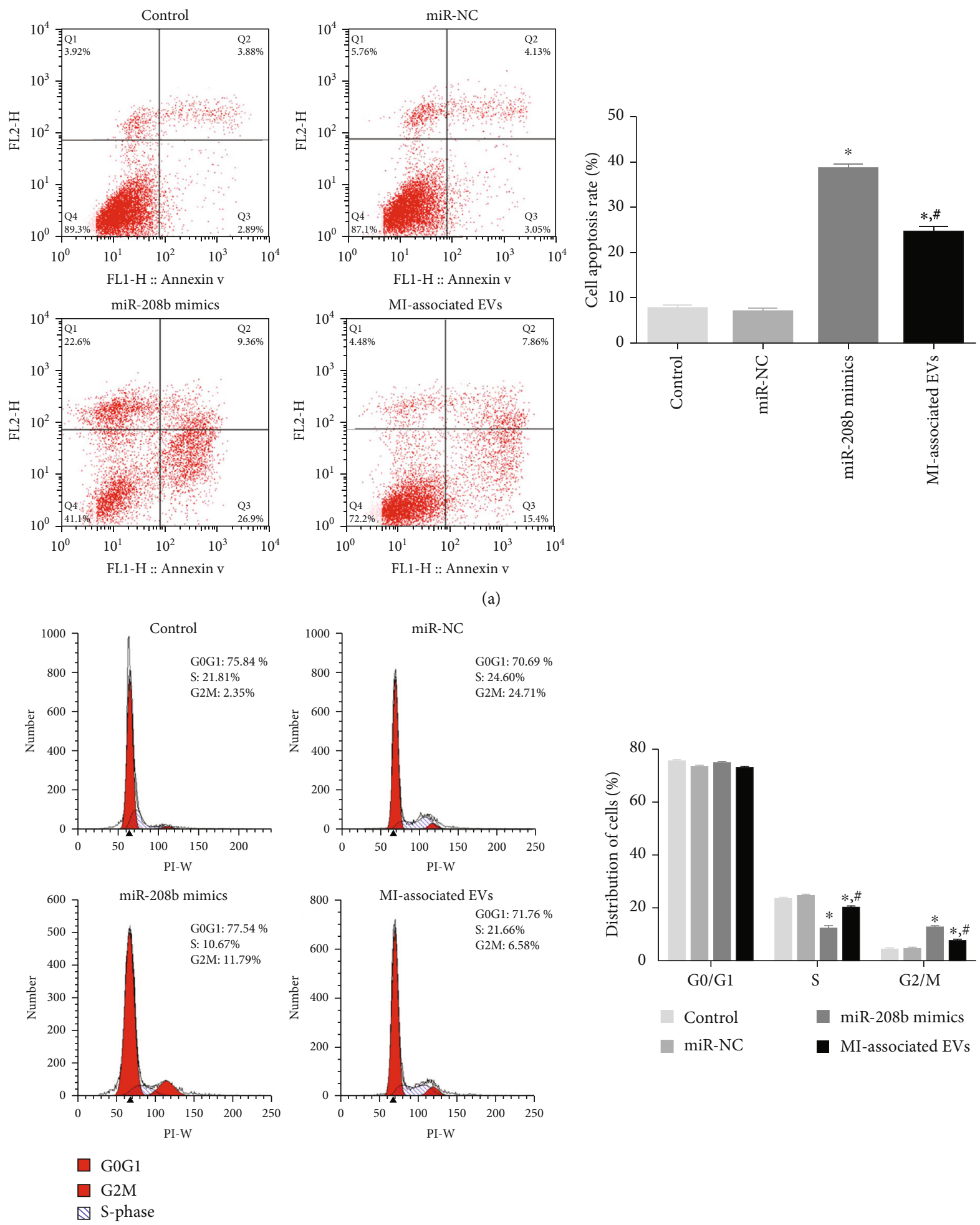

(b)

Figure 6: (a) The apoptosis rate of HUVECs in the different groups, determined by flow cytometry. Left: representative images acquired by flow cytometry. Right: apoptosis rates of the different groups, $n=3$. (b) The cycle progression of HUVECs following the different treatments, examined using flow cytometry. Left: images obtained by flow cytometry. Right: distribution of cells of the different groups in the different phases, $n=3$. ${ }^{*} P<0.05$, compared with the control group. ${ }^{\#} P<0.05$, compared with the miR-208b mimic group.

experiments. PKH67 staining subsequently demonstrated that EVs could be taken up by HUVECs, and the dualluciferase reporter gene assay further showed that CDKN1A was the target gene of miR-208b. The HUVECs were then transfected with either MI-associated exosomes or miR$208 \mathrm{~b}$ mimics, which demonstrated that both miR-208b 


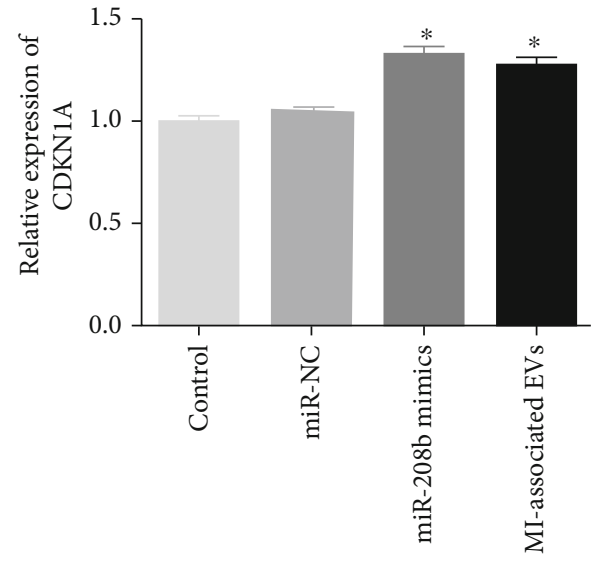

(a)

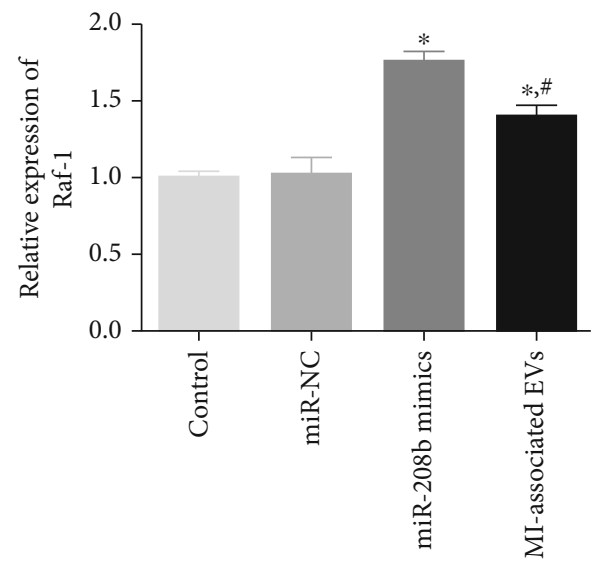

(c)

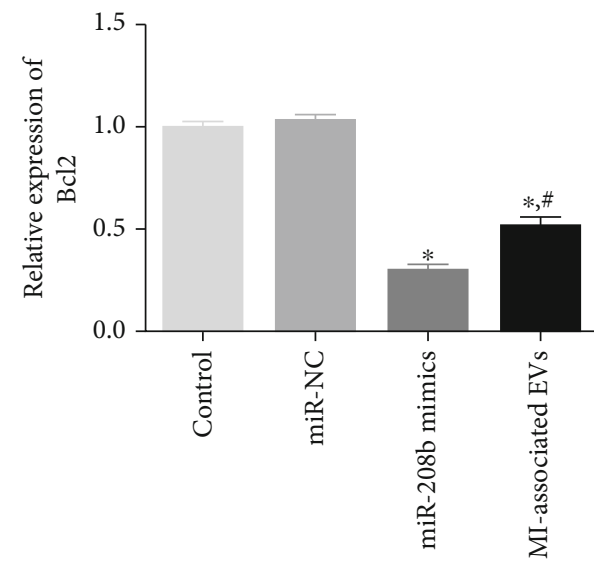

(e)

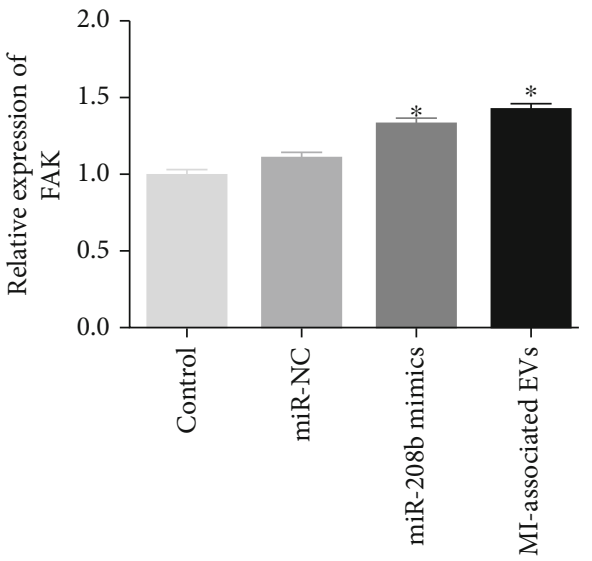

(b)

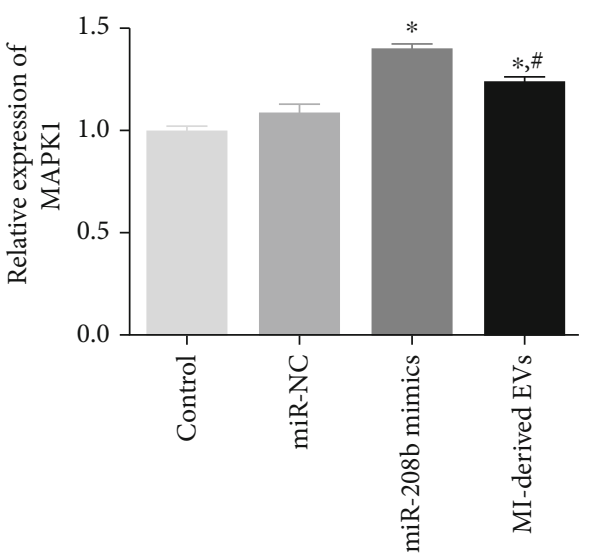

(d)

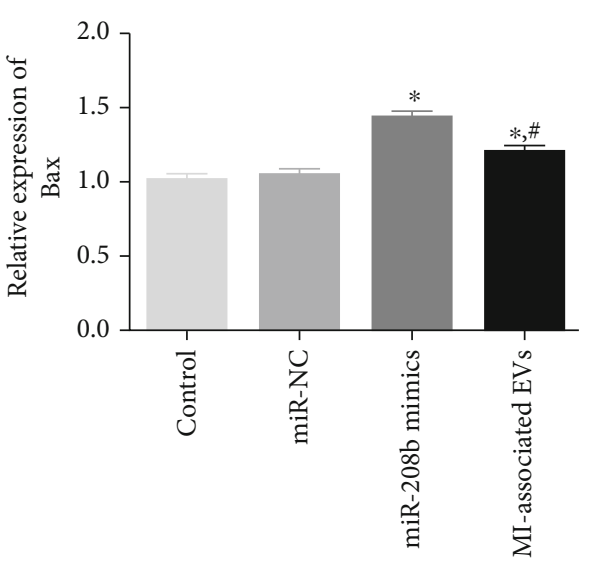

(f)

Figure 7: Continued. 


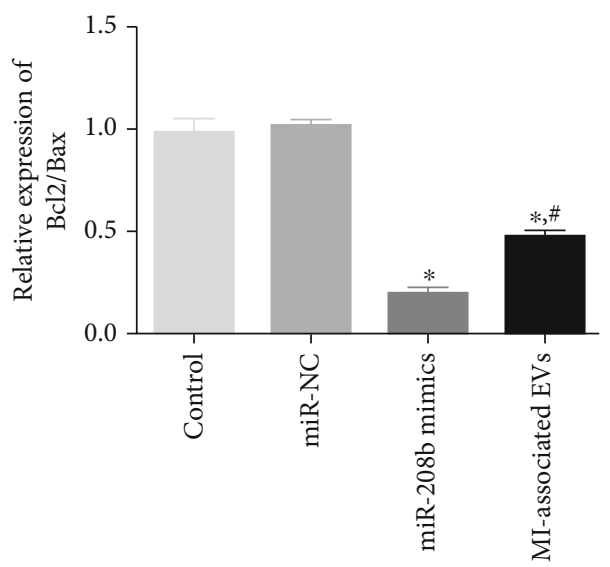

(g)

FIgURe 7: The expression levels of CDKN1A (a), $F A K(\mathrm{~b}), \operatorname{Raf}-1(\mathrm{c}), M A P K 1$ (d), $B c l 2$ (e), Bax (f), and Bcl2/Bax ratio (g) of the different groups, measured by RT-qPCR, $n=3 .{ }^{*} P<0.05$, compared with the control group. ${ }^{*} P<0.05$, compared with the miR-208b mimic group.

mimics and MI-associated EVs inhibited HUVEC viability and migration, promoted cell apoptosis, and led to decreased $S$ phase and increased G2/M phase cell distributions. Finally, RT-qPCR revealed that both miR-208b mimics and MIassociated EVs upregulated CDKN1A, FAK, Raf-1, MAPK1, and Bax but downregulated $B c l 2$ and reduced the Bcl2/Bax ratio. Our results suggested that MI-associated EVdelivered miR-208b may affect the growth of HUVECs by regulating apoptosis-related genes and CDKN1A, thereby influencing the progression of MI.

Previous studies have indicated that EVs serve as a natural carrier system that transport mRNA, miRNA, and proteins among cells and are involved in cardiovascular cell-to-cell communications [31, 32]. A study of Leroyer et al. [33] showed that endothelial cells release EVs enriched with miR$143 / 145$, which can be taken up by smooth muscle cells, and regulate gene expressions in the recipient cells, thus playing a role in atherosclerosis. Another study has shown that cardiac fibroblasts secrete EVs enriched in miR-21*, which can be shuttle to cardiomyocytes, and act as a key paracrine signaling mediator for cardiac hypertrophy [34]. Therefore, EVs can deliver miRNAs to other cells to perform their functions. MicroRNAs play key roles in MI by regulating apoptosis, necrosis, and autophagy. $\mathrm{Li}$ et al. [35] reported that miR208b was highly expressed in MI patients and that p21 was the direct target of miR-208b. In our study, miR-208b was also significantly enriched in MI patients than that in the healthy individuals, and miR-208b mimics exerted similar effects as MI-associated EVs. Both miR-208b mimics and MIassociated EVs suppressed HUVEC viability and migration and induced cell apoptosis. Additionally, they influenced the cell cycle by decreasing the distribution of the $S$ phase, while increasing that of the G2/M phase. A study by Zhou et al. [36] showed that miR-208b could alleviate heart injuries in MI rat models and inhibit postinfarction myocardial fibrosis, which implied that miR-208b may play an important role in MI. Another study demonstrated that exosomes isolated from adipose-derived stem cells with miR-126 enrichment prevented myocardial damage by protecting myocardial cells against apoptosis, inflammation, and fibrosis, as well as by promoting angiogenesis [37]. These findings, combined with our results, support the speculation that EVs can deliver miR-208b to HUVECs and that EV-delivered miR-208b may influence the development of MI by regulating cell viability, migration, apoptosis, and cell cycle events.

To further understand the molecular mechanisms to which MI-associated EVs and miR-208b affect HUVEC growth, the target gene of miR-208b was predicted, and the expressions of CDKN1A, FAK, Raf-1, MAPK1, Bcl2, and Bax were measured using RT-qPCR. By employing the online tool, TargetScan Human 7.1, and the dual-luciferase reporter gene assay, $C D K N 1 A$ was found to be the target gene of miR-208b; furthermore, RT-qPCR revealed that miR-208b mimics and $\mathrm{MI}$-associated EVs significantly upregulated CDKN1A expression. CDKN1A encodes for p21 protein and belongs to the Cip/Kip family [38]. CDKN1A is not only a tumor suppressor, a cell cycle inhibitor, and a senescence inducer but also a key regulator of cell migration, apoptosis, differentiation, DNA repair, and transcription $[39,40]$. In addition, CDKN1A is vital for G2/M transition and mitotic progression [41]. Our results showed that CDKN1A upregulation promoted cell apoptosis by decreasing the $S$ phase and increasing G2/M phase cell distributions. Gang et al. [42] indicated that $C D K N 1 A$ overexpression suppressed the proliferation and invasion of human fibroblast-like synoviocytes and arrested the cells in the G0/G1 phase. Another study has shown that CDKN1A overexpression increased the proportion of the G0/G1 phase cells, decreased that of the S phase cells, and elevated cell apoptosis in ovarian cancer cells [43]. These results suggested that EV-delivered miR-208b could affect cell apoptosis and cycle progression by mediating the miR-208b/CDKN1A pathway.

In addition, our study showed that transfection with either miR-208b mimics or MI-associated EVs significantly upregulated the expressions of FAK, Raf-1, MAPK1, and $B a x$, while significantly downregulating those of $B c l 2$ and significantly reducing the $B c l 2 / B a x$ ratio. $F A K$, an integrinassociated protein tyrosine kinase, plays a major role in cellular communication, especially in cellular signaling systems [44]. Previous studies have reported that FAK is 
overexpressed in many cancer cells and is closely associated with cell survival, proliferation, migration, and invasion $[45,46]$. Crowe and Ohannessian further demonstrated that $F A K$ regulated migration and invasion of squamous cell carcinoma lines by activating MPKAs [47]. MAPK1 is a key member of the MAPK/ERK pathway and participates in multifarious pathological processes of cells $[48,49]$, while Raf- 1 is a serine/threonine protein kinase that plays an important role in cell proliferation [50]. The Raf-1/MAPK1,3 pathway has been reported to be involved in the control of Bcl 2 family proteins and prevent the activation of Caspase 9 in the apoptosome complex [51]. Bcl2 is an antiapoptotic gene, while $B a x$ is a proapoptotic gene. Their ratio determines the trend of cell apoptosis, with $\mathrm{u}$-regulation of the $\mathrm{Bcl}-2 / \mathrm{Bax}$ ratio reflecting inhibited apoptosis and vice versa [52]. Findings of these studies, alongside ours, support the speculation that EV-delivered miR-208b might regulate apoptosis-related genes $(B c l 2$ and $B a x)$ via the FAK/MAPK1/Raf-1 pathway, thereby influencing HUVEC growth.

However, this study had several limitations. The number of clinical samples is small; therefore, our findings on the effects of miR-208b on CVD warrant further validation in larger samples and animal models and in vivo evaluation. The protein expressions of CDKN1A, Raf-1, FAK, Bcl2, MAPK1, p-MAPK1, and p-FAK should be analyzed using western blot, and the relationship of miR-208b with CDKN1A, Raf-1, FAK, Bcl2, Bax, and MAPK1 also require further verification by a series of rescue experiments. Additionally, the cell source of EVs extracted from the plasmas of MI patients and the factors contributing to the miR-208b enrichment in patients with acute MI need to be further explored.

\section{Conclusions}

In conclusion, MI-associated EVs can deliver miR-208b to HUVECs, and the enrichment of miR-208b may suppress cell viability and migration, as well as promote cell apoptosis by regulating apoptosis-related genes ( $\mathrm{Bcl} 2$ and $\mathrm{Bax}$ ) and the FAK/MAPK1/Raf-1 pathway. Additionally, EV-delivered miR-208b can induce cell apoptosis and cycle arrest in HUVECs by mediating the miR-208b/CDKN1A pathway. Our findings imply that EV-delivered miR-208b represents a promising target for the improvement of MI treatment, and targeting the miR-208b/CDKN1A pathway may function as a novel therapeutic approach in CVD.

\section{Data Availability}

The dataset used and/or analyzed during the current study are available from the corresponding author on reasonable request.

\section{Conflicts of Interest}

The authors declare that they have no competing interests.

\section{Authors' Contributions}

MW and WJ conceptualized, reviewed, edited, and supervised the study. WJ, QS, ZL, and SW developed the methodology and carried out the formal analyses. TL and XW carried out the investigations. WJ and MW prepared the original draft. All authors read and approved the final manuscript.

\section{Acknowledgments}

This study was supported by the Science and Technology Program of Hebei Province (13277729D).

\section{Supplementary Materials}

Supplementary Table S1: the physiological and biochemical indexes of myocardial infarction (MI) patients and healthy individuals. (Supplementary Materials)

\section{References}

[1] L. Lu, M. Liu, R. Sun, Y. Zheng, and P. Zhang, "Myocardial infarction: symptoms and treatments," Cell Biochemistry and Biophysics, vol. 72, no. 3, pp. 865-867, 2015.

[2] M. Kosuge, K. Kimura, T. Ishikawa et al., "Differences between men and women in terms of clinical features of st-segment elevation acute myocardial infarction," Circulation Journal, vol. 70, no. 3, pp. 222-226, 2006.

[3] M. Saleh and J. A. Ambrose, "Understanding myocardial infarction," F1000Res, vol. 7, article 1378, 2018.

[4] J. Li, X. Li, Q. Wang et al., "St-segment elevation myocardial infarction in China from 2001 to 2011 (the China peaceretrospective acute myocardial infarction study): a retrospective analysis of hospital data," Lancet, vol. 385, no. 9966, pp. 441-451, 2015.

[5] J. S. Hochman, L. A. Sleeper, J. G. Webb et al., "Early revascularization in acute myocardial infarction complicated by cardiogenic shock," The New England Journal of Medicine, vol. 341, no. 9, pp. 625-634, 1999.

[6] C. Théry, K. W. Witwer, E. Aikawa et al., "Minimal information for studies of extracellular vesicles 2018 (misev2018): a position statement of the international society for extracellular vesicles and update of the misev2014 guidelines," Journal of extracellular vesicles, vol. 7, no. 1, article 1535750, 2018.

[7] R. Kalluri, "The biology and function of exosomes in cancer," The Journal of Clinical Investigation, vol. 126, no. 4, pp. 1208-1215, 2016.

[8] C. Corcoran, A. M. Friel, M. J. Duffy, J. Crown, and L. O'Driscoll, "Intracellular and extracellular microRNAs in breast cancer," Clinical Chemistry, vol. 57, no. 1, pp. 18-32, 2011.

[9] S. A. Melo, L. B. Luecke, C. Kahlert et al., "Glypican-1 identifies cancer exosomes and detects early pancreatic cancer," Nature, vol. 523, no. 7559, pp. 177-182, 2015.

[10] J. Skog, T. Würdinger, S. van Rijn et al., "Glioblastoma microvesicles transport RNA and proteins that promote tumour growth and provide diagnostic biomarkers," Nature Cell Biology, vol. 10, no. 12, pp. 1470-1476, 2008.

[11] S. Gupta and A. A. Knowlton, "Hsp60 trafficking in adult cardiac myocytes: role of the exosomal pathway," American 
Journal of Physiology. Heart and Circulatory Physiology, vol. 292, no. 6, pp. H3052-H3056, 2007.

[12] B. W. van Balkom, O. G. de Jong, M. Smits et al., "Endothelial cells require miR-214 to secrete exosomes that suppress senescence and induce angiogenesis in human and mouse endothelial cells," Blood, vol. 121, no. 19, pp. 3997-4006, 2013.

[13] J. Halkein, S. P. Tabruyn, M. Ricke-Hoch et al., "MicroRNA146a is a therapeutic target and biomarker for peripartum cardiomyopathy," The Journal of Clinical Investigation, vol. 123, no. 5, pp. 2143-2154, 2013.

[14] Y. Feng, W. Huang, M. Wani, X. Yu, and M. Ashraf, "Ischemic preconditioning potentiates the protective effect of stem cells through secretion of exosomes by targeting Mecp2 via miR22," PLoS One, vol. 9, no. 2, article e88685, 2014.

[15] B. Yu, H. W. Kim, M. Gong et al., "Exosomes secreted from GATA-4 overexpressing mesenchymal stem cells serve as a reservoir of anti-apoptotic microRNAs for cardioprotection," International Journal of Cardiology, vol. 182, pp. 349-360, 2015.

[16] G. Bellin, C. Gardin, L. Ferroni et al., "Exosome in cardiovascular diseases: a complex world full of hope," Cells, vol. 8, no. 2, p. 166, 2019.

[17] B. Simonson and S. Das, "MicroRNA therapeutics: the next magic bullet?," Mini Reviews in Medicinal Chemistry, vol. 15, no. 6, pp. 467-474, 2015.

[18] A. Wojciechowska, A. Osiak, and K. Kozar-Kamińska, "MicroRNA in cardiovascular biology and disease," Advances in clinical and experimental medicine: official organ Wroclaw Medical University, vol. 26, no. 5, article 62915, pp. 868-874, 2017.

[19] K. Agiannitopoulos, P. Pavlopoulou, K. Tsamis et al., "Expression of miR-208b and miR-499 in greek patients with acute myocardial infarction," In Vivo, vol. 32, no. 2, pp. 313-318, 2018.

[20] C. Wang and Q. Jing, "Non-coding RNAs as biomarkers for acute myocardial infarction," Acta Pharmacologica Sinica, vol. 39, no. 7, pp. 1110-1119, 2018.

[21] R. Zhou, W. Huang, X. Fan et al., "miR-499 released during myocardial infarction causes endothelial injury by targeting (7-nAchR," Journal of Cellular and Molecular Medicine, vol. 23, no. 9, pp. 6085-6097, 2019.

[22] P. M. Vanhoutte, "Endothelial dysfunction: the first step toward coronary arteriosclerosis," Circulation Journal, vol. 73, no. 4, pp. 595-601, 2009.

[23] T. Soares Martins, J. Catita, I. Martins Rosa, O. da Cruz e Silva, and A. G. Henriques, "Exosome isolation from distinct biofluids using precipitation and column-based approaches," PLoS One, vol. 13, no. 6, article e0198820, 2018.

[24] Q. Zhu, Q. Li, X. Niu et al., "Extracellular vesicles secreted by human urine-derived stem cells promote ischemia repair in a mouse model of hind-limb ischemia," Cellular Physiology and Biochemistry, vol. 47, no. 3, pp. 1181-1192, 2018.

[25] J. Yin, A. Zeng, Z. Zhang, Z. Shi, W. Yan, and Y. You, "Exosomal transfer of miR-1238 contributes to temozolomideresistance in glioblastoma," eBioMedicine, vol. 42, pp. 238251, 2019.

[26] G. Xu, R. Ao, Z. Zhi, J. Jia, and B. Yu, "miR-21 and miR-19b delivered by hMSC-derived EVs regulate the apoptosis and differentiation of neurons in patients with spinal cord injury," Journal of Cellular Physiology, vol. 234, no. 7, pp. 1020510217, 2019.
[27] S. Sahoo, D. N. Meijles, I. Al Ghouleh et al., "MEF2C-MYOCD and leiomodin 1 suppression by miRNA-214 promotes smooth muscle cell phenotype switching in pulmonary arterial hypertension," PLoS One, vol. 11, no. 5, article e0153780, 2016.

[28] M. Tkach and C. Thery, "Communication by extracellular vesicles: where we are and where we need to go," Cell, vol. 164, no. 6, pp. 1226-1232, 2016.

[29] A. Saparov, V. Ogay, T. Nurgozhin et al., "Role of the immune system in cardiac tissue damage and repair following myocardial infarction," Inflammation Research, vol. 66, no. 9, pp. 739-751, 2017.

[30] J. Mills, M. Capece, E. Cocucci, A. Tessari, and D. Palmieri, "Cancer-derived extracellular vesicle-associated micrornas in intercellular communication: one cell's trash is another cell's treasure," International journal of molecular sciences, vol. 20, no. 24, p. 6109, 2019.

[31] C. Emanueli, A. I. Shearn, G. D. Angelini, and S. Sahoo, "Exosomes and exosomal miRNAs in cardiovascular protection and repair," Vascular Pharmacology, vol. 71, pp. 24-30, 2015.

[32] H. Valadi, K. Ekstrom, A. Bossios, M. Sjostrand, J. J. Lee, and J. O. Lotvall, "Exosome-mediated transfer of mRNAs and microRNAs is a novel mechanism of genetic exchange between cells," Nature Cell Biology, vol. 9, no. 6, pp. 654-659, 2007.

[33] A. S. Leroyer, H. Isobe, G. Leseche et al., "Cellular origins and thrombogenic activity of microparticles isolated from human atherosclerotic plaques," Journal of the American College of Cardiology, vol. 49, no. 7, pp. 772-777, 2007.

[34] Z. Giricz, Z. V. Varga, T. Baranyai et al., "Cardioprotection by remote ischemic preconditioning of the rat heart is mediated by extracellular vesicles," Journal of Molecular and Cellular Cardiology, vol. 68, pp. 75-78, 2014.

[35] C. Li, X. Chen, J. Huang, Q. Sun, and L. Wang, "Clinical impact of circulating miR-26a, miR-191, and miR-208b in plasma of patients with acute myocardial infarction," European Journal of Medical Research, vol. 20, no. 1, p. 58, 2015.

[36] C. Zhou, Q. Cui, G. Su, X. Guo, X. Liu, and J. Zhang, "MicroRNA-208b alleviates post-infarction myocardial fibrosis in a rat model by inhibiting GATA4," Medical science monitor: international medical journal of experimental and clinical research, vol. 22, article 896428, pp. 1808-1816, 2016.

[37] Q. Luo, D. Guo, G. Liu, G. Chen, M. Hang, and M. Jin, "Exosomes from miR-126-overexpressing ADSCs are therapeutic in relieving acute myocardial ischaemic injury," Cellular Physiology and Biochemistry, vol. 44, no. 6, pp. 2105-2116, 2017.

[38] N. N. Kreis, F. Louwen, and J. Yuan, "The multifaceted p21 $\left(\mathrm{Cip}_{1} / \mathrm{Waf}_{1} / C D K N 1 A\right)$ in cell differentiation, migration and cancer therapy," Cancers (Basel), vol. 11, no. 9, p. 1220, 2019.

[39] N. N. Kreis, F. Louwen, and J. Yuan, "Less understood issues: p $21^{\text {Cip1 }}$ in mitosis and its therapeutic potential," Oncogene, vol. 34, no. 14, pp. 1758-1767, 2015.

[40] V. S. Romanov and K. L. Rudolph, "P21 shapes cancer evolution," Nature Cell Biology, vol. 18, no. 7, pp. 722-724, 2016.

[41] N. N. Kreis, A. Friemel, B. Zimmer et al., "Mitotic p $21^{\text {Cip1/CD- }}$ $\mathrm{KN1A}$ is regulated by cyclin-dependent kinase 1 phosphorylation," Oncotarget, vol. 7, no. 31 , article 10330, pp. $50215-$ 50228, 2016.

[42] X. Gang, H. Xu, L. Si et al., "Treatment effect of CDKN1A on rheumatoid arthritis by mediating proliferation and invasion of fibroblast-like synoviocytes cells," Clinical and Experimental Immunology, vol. 194, no. 2, pp. 220-230, 2018. 
[43] H. Guo, C. Ha, H. Dong, Z. Yang, Y. Ma, and Y. Ding, "Cancer-associated fibroblast-derived exosomal microRNA-98-5p promotes cisplatin resistance in ovarian cancer by targeting CDKN1A," Cancer Cell International, vol. 19, no. 1, p. 347, 2019.

[44] B. Wang, X. Qi, D. Li, M. Feng, X. Meng, and S. Fu, "Expression of py397 FAK promotes the development of non-small cell lung cancer," Oncology Letters, vol. 11, no. 2, pp. 979983, 2016.

[45] J. Sun, Q. Luo, L. Liu, and G. Song, "Low-level shear stress promotes migration of liver cancer stem cells via the FAK-ERK1/2 signalling pathway," Cancer Letters, vol. 427, pp. 1-8, 2018.

[46] J. Zhou, Q. Yi, and L. Tang, "The roles of nuclear focal adhesion kinase (FAK) on cancer: a focused review," Journal of Experimental \& Clinical Cancer Research, vol. 38, no. 1, p. 250, 2019.

[47] D. L. Crowe and A. Ohannessian, "Recruitment of focal adhesion kinase and paxillin to $\beta 1$ integrin promotes cancer cell migration via mitogen activated protein kinase activation," BMC Cancer, vol. 4, no. 1, p. 18, 2004.

[48] J. Bai, Y. Zheng, G. Wang, and P. Liu, "Protective effect of Dlimonene against oxidative stress-induced cell damage in human lens epithelial cells via the p38 pathway," Oxidative Medicine and Cellular Longevity, vol. 2016, Article ID 5962832, 12 pages, 2016.

[49] L. K. Wu, Y. C. Liu, G. Ma, L. L. Shi, and X. M. He, "High levels of glucose promote the activation of hepatic stellate cells via the p38-mitogen-activated protein kinase signal pathway," Genetics and Molecular Research: GMR, vol. 15, no. 3, 2016.

[50] R. Wang, O. P. Mercaitis, L. Jia, R. A. Panettieri, and D. D. Tang, "Raf-1, actin dynamics, and abelson tyrosine kinase in human airway smooth muscle cells," American Journal of Respiratory Cell and Molecular Biology, vol. 48, no. 2, pp. 172-178, 2013.

[51] S. Cagnol, A. Mansour, E. Van Obberghen-Schilling, and J. C. Chambard, "Raf-1 activation prevents caspase 9 processing downstream of apoptosome formation," Journal of signal transduction, vol. 2011, Article ID 834948, 12 pages, 2011.

[52] K. Vucicevic, V. Jakovljevic, N. Colovic et al., "Association of Bax expression and $\mathrm{Bcl} 2 / \mathrm{Bax}$ ratio with clinical and molecular prognostic markers in chronic lymphocytic leukemia," Journal of medical biochemistry, vol. 35, no. 2, pp. 150-157, 2016. 